\title{
Marcha en personas con discapacidad visual: Revisión de literatura
}

\author{
Alemán-Ramírez, Carolina \\ Marcha en personas con discapacidad visual: Revisión de literatura \\ MHSalud, vol. 17, núm. 1, 2020 \\ Universidad Nacional, Costa Rica \\ Disponible en: http://www.redalyc.org/articulo.oa?id=237061117005 \\ DOI: https://doi.org/10.15359/mhs.17-1.5
}

Esta obra está bajo una Licencia Creative Commons Atribución-NoComercial-SinDerivar 3.0 Internacional. 


\section{Marcha en personas con discapacidad visual: Revisión de literatura}

\author{
Gait of Individual With Visual Impairment: Literature Review \\ Caminhada no deficiente visual: Revisão da literatura \\ Carolina Alemán-Ramírez \\ Universidad Nacional, Costa Rica \\ caroaleman@gmail.com
}

DOI: https://doi.org/10.15359/mhs.17-1.5
Redalyc: http://www.redalyc.org/articulo.oa?
id $=237061117005$

Recepción: 19 Septiembre 2019

Aprobación: 22 Noviembre 2019

\section{ReSUMEN:}

Introducción: Caminar para las personas con discapacidad visual (PDV) presenta diversas variaciones que afectan su funcionalidad y su salud, especialmente, porque el impacto al sistema músculo esquelético. Propósito: realizar una revisión de literatura para analizar el patrón de la caminata, así como las implicaciones que esta tiene en individuos de baja visión y ceguera. Método: para la obtención de información de la revisión de literatura sistemática, se buscaron artículos científicos en las bases de datos que a continuación se mencionan: SportDiscus con texto completo, psycINFO, Psychology and Behavioral Sciences Collection, Rehabilitation \& Sports Medicine Source, google académico, Research Gate, PubMed. Resultados: las PDV, muestran una caminata cautelosa, probablemente para protegerse de las barreras arquitectónicas a las que podrían enfrentarse. Conclusiones: las PDV, presentan movimientos más cortos, lentos, lo que indica menores rangos de movimiento, afectando su funcionalidad, y generando un estrés muscular. Es muy importante la valoración de la caminata en las PDV, para que se desarrollen patrones adecuados y no presenten desbalances o acortamientos musculares. Logrando una mejora de su locomoción y con esta una mayor independencia.

Palabras Clave: marcha, locomoción, baja visión, ceguera.

\section{Abstract:}

Introduction: The gait in people with visual impairment (PWVI) has several variations that affect functionality and health, especially because it affects the skeletal muscle system. Purpose: to conduct a literature review to analyze the gait pattern and the implications it has on individuals with low vision and blindness. Method: a systematic review was held in the following databases: SportDiscus with full text, PsycINFO, Psychology, and Behavioral Sciences Collection, Rehabilitation \& Sports Medicine Source, Google Scholar, Research Gate, PubMed. Results: The way of walking is slower in PWVI; they are cautious when walking to protect themselves, although the surrounding is clear. The disadvantage of this variable is that people do not walk in accordance with the optimal level of walking according to their age and height (recommended speed), so there would be no economy of movement; besides, its functionality is also affected. Conclusions: The research showed that the PWVI had atypical gait patterns. It was found, in some cases, greater ranges of movement in the involved joints, or on the diminished contrary movements, indicating small or forced movements, unnatural and, therefore, greater stress on muscle and bone. Evaluations in real situations on PWVI are very important to be able to provide them with tools that can help them in their walking and accessibility.

KEYWORDS: Gait, visually impaired, locomotion, blindness, walking.

\section{Resumo:}

Introdução: A marcha para pessoas com deficiência visual (PDV) tem várias variações que afetam a funcionalidade e a saúde, especialmente porque impacta o sistema muscular esquelético. Objetivo: Realizar uma revisão da literatura para analisar o padrão de marcha e as implicações que ela tem nos indivíduos com baixa visão e cegueira. Método: a fim de obter informações da revisão sistemática da literatura, os artigos científicos foram pesquisados nas seguintes bases de dados: SportDiscus com texto completo, PsycINFO, Psychology, and Behavioral Sciences Collection, Rehabilitation \& Sports Medicine Source, Google Scholar, Research Gate, PubMed. Resultados: os PDVs mostram uma caminhada cautelosa, provavelmente para se protegerem das barreiras arquitetônicas que podem enfrentar. Conclusões: A PDV apresenta movimentos mais curtos e lentos, o que indica menores intervalos de movimento, afetando sua funcionalidade e gerando estresse muscular. É muito importante a avaliação da marcha no PDV, para que padróes adequados sejam desenvolvidos e não apresentem desequilíbrios ou encurtamentos musculares, conseguindo uma melhora de sua locomoção e com isso uma maior independência.

Palavras-CHAVE: marcha, locomoção, baixa visão, cegueira. 


\section{INTRODUCCIÓN}

Al menos el $30 \%$ de las personas con discapacidad visual (PDV), no son independientes en el exterior de sus hogares, esto podría deberse a diferentes causas, como falta de: recursos económicos, oportunidades, motivación (Clark-Carter, Heyes y Howarth, 1986). Del mismo modo, la autonomía se puede ver afectada por limitadas habilidades en sus desplazamientos, en la orientación, en la capacidad de subir y bajar gradas, y en la forma y la velocidad de caminar. Ahora bien, centrándonos en este patrón de movimiento, caminar es un elemento esencial para la capacidad de independencia y movilidad, su ausencia o no ejecución influye directamente en los bajos niveles de actividad física (Aydoğ et al., 2006; Chen y Lin, 2011; Chen et al., 2012; Kobberling, Jankowski, y Leger, 1989; Larsson y Frändin, 2006).

Actualmente, se cuenta con ayudas como el uso del bastón, que facilitan las caminatas en PDV, porque les permite detectar cualquier tipo de barrera arquitectónica que se presente (huecos, obstáculos, aceras, bordes, postes), sin embargo, es necesario adquirir o mejorar habilidades y destrezas motoras de forma prioritaria que propicien la eficiencia en su desplazamiento, mejorando su funcionalidad, para luego aprender a utilizar elementos o ayudas que mejoren su movilidad independiente (Clark-Carter, Heyes y Howarth, 1986; Alan 1991; Theodorou y Skordilis, 2012; Żyłka y Rutkowska, 2013, Reynard y Terrier, 2015; White et al., 2015; Parreira, Grecco y Oliveira, 2017).

\section{La relación de la visión con la locomoción}

La locomoción, entendida como el traslado de un lugar a otro, permite realizar labores en la vida cotidiana, contribuye en la independencia y la funcionalidad del ser humano, además, asiste en necesidades como la búsqueda de alimento y el acceso a lugares específico (Patla, 1997; Larsson y Frändin, 2006; Hallemans, Ortibus, Meire y Aerts, 2010).

Ahora bien, la locomoción se encuentra en parte regulada por el sistema visual, en aspectos como los siguientes: mantenimiento de la estabilidad corporal, orientación espacial, planeación de rutas, información del ambiente, distancias y obstáculos, por lo que permite acomodar el cuerpo en diferentes superficies (Patla, 1997; Saibene y Minetti, 2003; Hallemans et al., 2010).

Uno de los objetivos de la percepción visual durante la locomoción, es que la visión permite acomodar los movimientos de las extremidades, ajustando el movimiento, es así como se logra esquivar obstáculos cambiando de dirección, se prepara el cuerpo para variantes en desniveles, o incluso se regula la velocidad de la marcha (Hallemans et al., 2010).

Del mismo modo, Patla (1997), menciona que el sistema visual cumple con varias funciones en el control y la estabilidad de la locomoción, tales como:

1. Establecer la postura inicial necesaria.

2. Iniciar y terminar la locomoción cuándo y cómo sea necesario.

3. Producir y coordinar la activación rítmica de las extremidades y del tronco para impulsar al cuerpo en la dirección prevista.

4. Mantener la estabilidad dinámica del movimiento del cuerpo, contrarrestando la fuerza de gravedad.

5. Mantener o alterar la velocidad para evitar obstáculos, colocar y acomodar el pie estable en diferentes terrenos o cambios de dirección.

6. Proteger la estabilidad estructural.

De igual manera, Agostini et al. (2016), mencionan tres teorías relacionadas con la visión central y periférica; la primera es la "teoría de la dominación periférica", que enfatiza que la visión periférica, tiene un 
rol en el control de la postura, la segunda es conocida como la "hipótesis de invariancia retiniana" y sugiere que la visión central y periférica tiene el mismo papel funcional, para mantener una postura erguida y la tercera teoría "hipótesis de sensibilidad funcional", sostiene que existen diferencias en los roles funcionales y complementarios para la visión central y periférica en el control postural, en particular, argumenta que la visión periférica es predominante en el control postural antero-posterior (AP), mientras que la visión central en el medio-lateral (ML).

Por lo anterior, podríamos decir que la visión es entonces, una clave fundamental para la ejecución de la locomoción, la postura y la caminata (Gazzellini et al., 2016).

\section{Aspectos que se involucran en la caminata}

Agudelo et al. (2013), mencionan que la caminata es la unidad esencial del movimiento, es un elemento básico y complejo, es una herramienta que aumenta las posibilidades motoras, además, facilita la interacción social y la exploración del ambiente. Existen diferentes factores que alteran la forma de caminar, ya sea de manera temporal o permanente, como lo son:

- Patológicos (traumatismos, neurológicos, músculo esqueléticos o trastornos psiquiátricos).

- Psicológicos (relacionados con la personalidad y las emociones del individuo).

- Fisiológicos (periodo de gestación, proceso normal de envejecimiento).

- Físicos (medidas antropométricas).

- Factores extrínsecos (terreno, tipo de calzado, vestido,) e intrínsecos (edad, sexo).

Ahora bien, la marcha tiene dos fases, la de soporte o de apoyo, que compone un $60 \%$ (contacto inicial, respuesta a la carga, soporte medio, soporte terminal, prebalanceo) y la de balanceo que ocupa el $40 \%$ (balanceo inicial, medio y final).

Ramsey et al. (1999) y Knutzen, Hamill \& Bates (1985), mencionan los parámetros de la caminata: espaciales (longitud de zancada y de paso, ancho del paso, altura del paso, ángulo del paso); temporales (apoyo, balanceo, doble apoyo, periodo de zancada, periodo de soporto, periodo de balanceo, cadencia), y espaciotemporales (velocidad, cadencia, velocidad de balanceo).

También, se ha clasificado en dos grandes tipos: la caminata o la marcha y la carrera. La caminata hace posible largos periodos de tiempo con movimientos lentos, mientras que la carrera no permitiría estos tiempos de duración tan prolongados (Saibene \& Minetti, 2003).

En la marcha el elemento de orientación pertenece al sistema sensorial y vestibular, y la movilidad pertenece al sistema motor, ambos necesitan de la visión para un buen funcionamiento, y así lograr percibir los cambios de dirección por las obstrucciones en el camino y tomar decisiones, también, para adaptar la postura. En el caso de las PDV para que puedan caminar con seguridad y eficientemente, deben de utilizar una combinación somatosensorial (Nakamura, 1997).

Después de la literatura expuesta anteriormente, se plantea como propósito realizar una revisión de literatura para conocer las características de la caminata adoptada en las personas de baja visión o ceguera, por tanto, con esta información se podría detectar elementos importantes para mejorar las posibles deficiencias, contando con evidencia científica, que permita un abordaje efectivo en su mejoramiento.

\section{Justificación}

Al evaluar la caminata, se cuenta con una herramienta diagnóstica importante, relacionada con el sistema músculo esquelético; su análisis permite apreciar diversos factores que pueden ayudar a la modificación, contando con el control y el seguimiento de un tratamiento, para lograr corregir diferentes aspectos, lo que 
contribuiría con la independencia y la funcionalidad del individuo. Además, es la primera revisión del tema, lo que permite resumir, resaltar y concretar aspectos por tomar en cuenta de la caminata en PDV.

\section{Metodología}

En la aplicación de la metodología se utilizaron, los lineamientos PRISMA (Moher, Liberati, Tetzlaff, y Altman 2009).

\section{Criterios de inclusión}

Los criterios de inclusión para la elaboración de la revisión fueron los siguientes:

- Publicaciones en idioma inglés o español.

- Sin restricción de fechas de inclusión en los artículos.

- Presentar texto completo.

- Medición en personas con discapacidad visual.

- Estudios cuasiexperiementales y experimentales.

- Evaluación de la marcha con diferentes métodos.

\section{Búsqueda de literatura}

En el proceso de la selección de los artículos del estudio, se realizó una búsqueda sin restricción de años en las bases de datos: EBSCOHost (Academic Search Ultimate, Behavioral Sciences Collection, SportDiscus, psycINFO, Psychology and, Rehabilitation \& Sports Medicine Source), google académico, PubMed. Para llevar a cabo esta búsqueda de referencias se utilizaron las siguientes frases boleanas: gait and visually impaired, blindness and walking, blind individuals and evaluation biomechanics, blindness. La Figura 1, presenta el diagrama de flujo de la búsqueda de revisión de literatura.

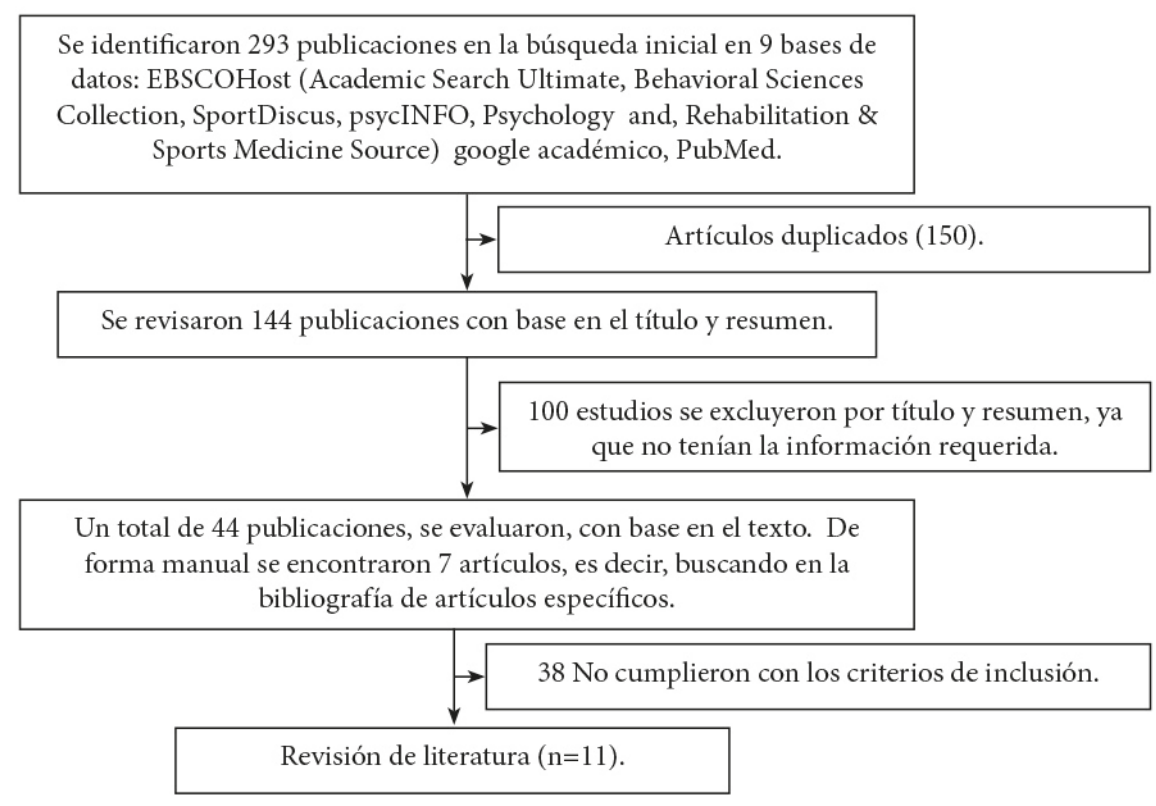

FIGURA 1

Diagrama de flujo del proceso de selección de información. Elaboración propia. 


\section{Resultados}

A continuación, se presenta la Tabla 1, con un resumen de los estudios encontrados, que analiza la marcha en personas con deficiencia visual.

TABLA 1

Resumen de artículos

\begin{tabular}{|c|c|c|c|c|c|c|}
\hline $\begin{array}{l}\text { Autor } y \\
\text { año }\end{array}$ & Sujetos/edad & $\begin{array}{l}\text { Tipo de equipo o } \\
\text { prueba utilizado }\end{array}$ & $\begin{array}{c}\text { Parámetros de la } \\
\text { caminata }\end{array}$ & Tarea & Objetivo del estudio & Resultados generales \\
\hline $\begin{array}{l}\text { Ray et ál. } \\
(2007)\end{array}$ & $\begin{array}{l}15 \text { PDV }(38.07 \pm 13.40 \\
\text { años), } \\
15 \text { VN }(38.13 \pm 131.16 \\
\text { años). }\end{array}$ & $\begin{array}{l}\text { Plataforma de } \\
\text { fuerza. }\end{array}$ & $\begin{array}{l}\text { Centro de gravedad. } \\
\text { Velocidad. }\end{array}$ & $\begin{array}{l}\text { Sentarse y levantarse, } \\
\text { caminar. }\end{array}$ & $\begin{array}{l}\text { Comparación entre } \\
\text { grupos. }\end{array}$ & $\begin{array}{l}\text {-Las PDV son más cautelosas, presentan dificultad } \\
\text { de realizar tareas si su centro de gravedad esta } \\
\text { fuera de su base de apoyo, por lo que tratan de no } \\
\text { realizar amplitud en sus movimientos. } \\
\text {-PDV caminaban más despacio, a pesar, que su } \\
\text { edad promedio era de } 38 \text { años, su ritmo fue el de } \\
\text { adultos mayores }(3 \mathrm{~km} / \mathrm{h}) \text {. }\end{array}$ \\
\hline $\begin{array}{l}\text { Nakamura, } \\
\text { (1997) }\end{array}$ & $\begin{array}{l}15 \text { hombres PDV ( } 44,3 \\
\text { años), 15 VN }(43,8 \\
\text { años) }\end{array}$ & $\begin{array}{l}\text { Sistema de } \\
\text { analizador de } \\
\text { movimiento } \\
\text { (NAC, modelo } \\
\text { HSV-400). }\end{array}$ & $\begin{array}{l}\text { GV: velocidad de la } \\
\text { marcha. } \\
\text { SL: longitud de la } \\
\text { zancada. } \\
\text { Tiempo en la fase de } \\
\text { marcha. }\end{array}$ & $\begin{array}{l}\text { Caminar descalzos en } 10 \mathrm{~m} \text {, } \\
\text { con marcadores reflectivos } \\
\text { en articulaciones. }\end{array}$ & $\begin{array}{l}\text { Comparación entre } \\
\text { grupos. }\end{array}$ & $\begin{array}{l}\text { Las PDV caminaron más despacio, con una } \\
\text { longitud de zancada más corta. } \\
\text { Caminar despacio podría deberse a la adaptación } \\
\text { del equilibrio dinámico reducido. } \\
\text { Las PDV responden a un control anticipatorio en } \\
\text { respuesta a cambios del entorno. }\end{array}$ \\
\hline $\begin{array}{l}\text { Ramsey, et } \\
\text { ál. (1999) }\end{array}$ & $\begin{array}{l}18 \text { hombres } \\
2 \text { mujeres } \\
\text { (50 } \pm 14,9 \text { años). }\end{array}$ & $\begin{array}{l}\text { Sistema de captura } \\
\text { (análisis de } \\
\text { movimiento, santa } \\
\text { rosa, CA). }\end{array}$ & $\begin{array}{l}\text { GV: velocidad de } \\
\text { caminata. } \\
\text { SL: longitud de la } \\
\text { zancada. } \\
\text { SR: ritmo de paso. }\end{array}$ & $\begin{array}{l}\text { Caminar en una pasarela de } \\
2.44 \times 3.65 \text { metros. }\end{array}$ & $\begin{array}{l}\text { Conocer los cambios } \\
\text { de la caminata, en } \\
\text { diferentes aspectos. } \\
\text { WN: caminata normal. } \\
\text { AD: caminata de bajada. } \\
\text { ST: caminar mientras } \\
\text { responde a una tarea } \\
\text { audible. } \\
\text { STAD: caminando } \\
\text { una bajada simulada y } \\
\text { responde a una tarea } \\
\text { audible. }\end{array}$ & \begin{tabular}{|l}
$\mathrm{GV}=\mathrm{WN} \uparrow \mathrm{ST} \uparrow$ \\
$\mathrm{SL}=\mathrm{WN} \uparrow$ \\
Hay diferencias en el ciclo de la caminata, según la \\
atención de la tarea. \\
Se encontró que la velocidad y la longitud de \\
zancada fueron más grandes en la condición \\
de caminata normal y en la caminata mientras \\
responde a una tarea audible. \\
Se concluye que hay diferencias en el ciclo de la \\
caminata de acuerdo a la atención de la tarea.
\end{tabular} \\
\hline $\begin{array}{l}\text { Mason, } \\
\text { Legge, } \\
\text { \& KRllie, } \\
\text { (2005) } \\
\end{array}$ & $\begin{array}{l}6 \mathrm{VN}(56,5 \text { años }) \\
12 \mathrm{DV}(27,3 \text { años). }\end{array}$ & $\begin{array}{l}\text { Podómetro } \\
\text { Pruebas de campo, } \\
\text { en una pista de } \\
\text { atletismo. }\end{array}$ & $\begin{array}{l}\text { Cambios de velocidad } \\
\text { en caminata. } \\
\text { Número de pasos. }\end{array}$ & $\begin{array}{l}\text { Caminar con cambios de } \\
\text { ritmo. }\end{array}$ & $\begin{array}{l}\text { Comparación entre } \\
\text { grupos. }\end{array}$ & $\begin{array}{l}\text { Cuando se escogió el ritmo, la longitud de paso } \\
\text { fue la más corta. Las PDV variaron su patrón } \\
\text { de caminata dependiendo de la velocidad, } \\
\text { comparado con VN. }\end{array}$ \\
\hline $\begin{array}{l}\text { Larsson \& } \\
\text { Frändin, } \\
\text { (2006) }\end{array}$ & 8 PDV (30- 62 años). & Pruebas de campo. & $\begin{array}{l}\text { Longitud del paso. } \\
\text { Velocidad en } \\
\text { caminata. }\end{array}$ & $\begin{array}{l}\text { Realizaron pruebas: } \\
\text { longitud del paso (MLS). } \\
\text { "Timed Up and Go" (TUG). } \\
10 \text { metros a máxima } \\
\text { velocidad. }\end{array}$ & $\begin{array}{l}\text { Conocer el efecto de un } \\
\text { tratamiento de danza } \\
\text { en el equilibrio y en la } \\
\text { velocidad de la marcha, } \\
\text { durante } \\
8 \text { semanas de } \\
\text { tratamiento, } 2 \text { veces por } \\
\text { semana. }\end{array}$ & $\begin{array}{l}\text { Mejoraron la velocidad del desplazamiento y el } \\
\text { balance. }\end{array}$ \\
\hline $\begin{array}{l}\text { Halleman, } \\
\text { et ál. (2010) }\end{array}$ & $\begin{array}{l}10 \mathrm{PDV} \\
(26.7 \pm 12.7 \text { años }) \\
20 \mathrm{VN} \\
\text { ( } 28.1 \pm 6.6 \text { años). }\end{array}$ & $\begin{array}{l}\text { Sistema } \\
\text { automatizado de } \\
\text { cámara infrarroja. } \\
\text { Marcadores } \\
\text { en puntos } \\
\text { anatómicos. } \\
2 \text { plataformas de } \\
\text { fuerza. }\end{array}$ & $\begin{array}{l}\text { Ángulo de la cabeza. } \\
\text { Velocidad de } \\
\text { caminata. } \\
\text { Largo del paso. } \\
\text { Cadencia. }\end{array}$ & $\begin{array}{l}\text { Caminaron en una pasarela } \\
\text { de } 1,5 \times 11 \mathrm{~m} \text {. }\end{array}$ & $\begin{array}{l}\text { Tres grupos } \\
\text { FV: visión } \\
\text { NV: no visión } \\
\text { VI: Discapacidad visual }\end{array}$ & $\begin{array}{l}\text { Diferencias entre } \\
\text { ángulo de la cabeza: NV y FV } \\
\text { Velocidad de caminata: } \\
\text { NV y FV } \\
\text { Largo del paso } \\
\text { VI y FV } \\
\text { NV y FV } \\
\text { Cadencia } \\
\text { NV y FV } \\
\text { NV y VI } \\
\text { La locomoción de PDV se adapta a pesar de que } \\
\text { exista un entorno despejado, por lo que realizan } \\
\text { una caminata más lenta y cautelosa, además, } \\
\text { realizaron exploración del terreno utilizando el pie. }\end{array}$ \\
\hline $\begin{array}{l}\text { (Uysal } \\
\text { et al., 2010) }\end{array}$ & 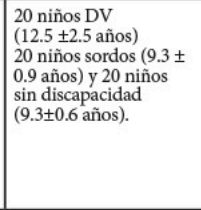 & $\begin{array}{l}\text { Cronómetro. } \\
\text { Podómetro } \\
\text { Goniómetro. } \\
\text { Pruebas de campo. }\end{array}$ & $\begin{array}{l}\text { Longitud de zancada. } \\
\text { Longitud del paso. } \\
\text { Ancho del paso. } \\
\text { Angulo del pie. } \\
\text { Pasos por minuto. } \\
\text { y velocidad de } \\
\text { caminata. }\end{array}$ & $\begin{array}{l}\text { Caminaron una plataforma } \\
\text { oscura. }\end{array}$ & $\begin{array}{l}\text { Comparación de los tres } \\
\text { grupos. }\end{array}$ & $\begin{array}{l}\text { Niños con ceguera giraron los pies hacia afuera, } \\
\text { con pasos mass pequeños y lentos. } \\
\text { La falta de visión afecta el desarrollo motor, } \\
\text { porque se les dificulta el movimiento libre y el } \\
\text { desarrollo de orientación espacial. } \\
\text { Se recomienda realizar estudios con muestras } \\
\text { de diversos antecedentes socioeconómicos y } \\
\text { culturales, ya que estas dos variables familiares y } \\
\text { sociales pueden influir en el proceso de desarrollo } \\
\text { en niños con discapacidad auditiva y visual. }\end{array}$ \\
\hline $\begin{array}{l}\text { Timmis, et } \\
\text { ál. (2014) }\end{array}$ & $\begin{array}{l}10 \mathrm{PDV} \\
\text { (77 } \pm 10 \text { años) } \\
13 \mathrm{VN} \\
\text { (72 } \pm 6 \text { años). }\end{array}$ & $\begin{array}{l}\text { Sistema de captura } \\
\text { de movimiento } \\
\text { de } 6 \text { cámaras, } \\
\text { y marcadores } \\
\text { reflectivos. }\end{array}$ & $\begin{array}{l}\text { Velocidad de } \\
\text { caminata. } \\
\text { Subir gradas. }\end{array}$ & $\begin{array}{l}\text { Subir gradas con diferentes } \\
\text { alturas: } \\
8.5 \mathrm{~cm} \\
16 \mathrm{~cm} \\
23.5 \mathrm{~cm}\end{array}$ & $\begin{array}{l}\text { Comparación PCV y } \\
\text { VN. }\end{array}$ & $\begin{array}{l}\text { PDV duraron más tiempo de contacto en el } \\
\text { escalón, lo que reduce el riesgo de contacto al } \\
\text { borde, mayor flexión de cabeza (un enfoque } \\
\text { más hacia abajo, más tiempo en el inicio de la } \\
\text { caminata, además se mostraron más cauteloso } \\
\text { (más tiempo en la planificación de la marcha) } \\
\text { entre más alta las gradas. Hay mayor variabilidad } \\
\text { en la marcha para adaptarse a los cambios de } \\
\text { terrenos lo que podría indicar mayor riesgo de } \\
\text { caerse. }\end{array}$ \\
\hline $\begin{array}{l}\text { Gazzellini } \\
\text { et ál. (2016) }\end{array}$ & $\begin{array}{l}12 \text { niños DV }(7 \pm 3 \\
\text { años) } \\
11 \text { niños VN }(8.3 \pm 2.8 \\
\text { años). }\end{array}$ & $\begin{array}{l}\text { Plataforma de } \\
\text { fuerza y una } \\
\text { cámara de captura } \\
\text { de movimiento } \\
\text { “optoelectronic } \\
\text { system". }\end{array}$ & $\begin{array}{l}\text { Velocidad de } \\
\text { caminata. } \\
\text { Ancho de paso. } \\
\text { Ángulo de tobillos. } \\
\text { Movimiento de } \\
\text { cabeza. }\end{array}$ & $\begin{array}{l}\text { Caminar de forma libre en } \\
\text { un espacio de } 7 \times 14 \mathrm{~m} .\end{array}$ & $\begin{array}{l}\text { Comparación de los } \\
\text { grupos. }\end{array}$ & $\begin{array}{l}\text { Los niños DV presentaron: velocidad disminuida, } \\
\text { paso más ancho, más duración de doble soporte } \\
\text { y rotación externa del ángulo del pie, fuerza de } \\
\text { reacción del suelo reducida, momento y potencia } \\
\text { en la postura tardia, aumento en la flexión de } \\
\text { la cabeza y disminución de la anteversión de la } \\
\text { pelvis, en comparación con el control grupo. }\end{array}$ \\
\hline $\begin{array}{l}\text { Da Silva } \\
\text { et ál. (2018) }\end{array}$ & 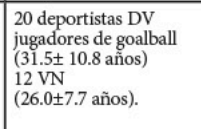 & $\begin{array}{l}\text { Prueba de campo: } \\
\text { indice de } \\
\text { rehabilitación } \\
\text { locomotora (LRI). }\end{array}$ & $\begin{array}{l}\text { Velocidad de zancada. } \\
\text { Ritmo de caminata. }\end{array}$ & Caminaron $15 \mathrm{~m}$. & $\begin{array}{l}\text { Comparación entre } \\
\text { grupos. }\end{array}$ & $\begin{array}{l}\text { Mencionan que por lo general las PDV, caminan } \\
\text { con un costo metabólico alto, sin embargo, en } \\
\text { este estudio los deportistas con DV tienen una } \\
\text { velocidad de marcha similar a personas VN; las } \\
\text { PDV sí mostraron mayor miedo de sufrir alguna } \\
\text { caída. }\end{array}$ \\
\hline
\end{tabular}




\section{Discusión}

En los estudios analizados, se encontraron diferencias en los parámetros de caminata entre las PDV y PVN. Las PDV caminan de manera más cautelosa y lenta, porque al no conocer el entorno al que se enfrentan, ya sean desniveles, obstáculos de diferentes alturas, se protegen en los traslados, haciendo adaptaciones como el caso de la longitud del paso y velocidad (Clark-Carter et al., 1986; Chen et al., 2012; da Silva et al., 2018). Un inconveniente de la reducción de la velocidad de la marcha es que las personas no aprovechan su nivel óptimo de caminata, es decir, que no la realizan de acuerdo a la edad, y a la longitud de las piernas, dando como resultado poca o baja economía de movimiento, como lo mencionan Ray et al. (2007), en donde PDV de 38 años caminaban tan despacio como adultos mayores de entre $70-90$ años, a una velocidad de $3 \mathrm{~km} /$ $\mathrm{h}$, es decir se da una afectación al sistema músculo esquelético, influyendo en la funcionalidad, así como también podría darse un incremento del dolor por sobrecarga. Con respecto a los movimientos cautelosos o reservados de las extremidades, se puede decir, que las PDV realizan acciones más controladas, cerca del centro de gravedad, porque perciben un mayor control corporal, lo que les brinda seguridad en sus desplazamientos (Patla, 1997; Larsson y Frändin, 2006; Uysal et al., 2010; Agudelo et al., 2013). Nakamura (1997), menciona que algunas de las estrategias para aumentar la velocidad y la eficiencia de la caminata podrían ser: caminar con un acompañante o un perro guía, hacer uso del bastón; mejorando así también la postura, la estabilidad y la seguridad.

Cuando las PDV se enfrentaron a situaciones como subir gradas o realizar cambios de dirección, propiciaron adaptaciones a la caminata como mayor duración en el apoyo de doble soporte, es decir, más tiempo con los pies juntos; una rotación externa del pie, lo que brinda un apoyo más amplio; un control anticipatorio, referente a la exploración o adelantamiento del pie para saber que hay adelante en su recorrido (aumentando, el ángulo de extensión del tobillo), logrando así, protección a la integridad física. También, se puede mencionar que, al aumentar la dificultad de la caminata, ya sea con sonidos ambientales o con la resolución de tareas, el ritmo disminuye al compararlo con un traslado en línea recta y sin obstáculos (Larsson \& Frändin 2006; Timmis et al., 2014).

Otras de las adaptaciones de las PDV cuando caminan son: mayor flexión de tronco y de cabeza, mínimo movimiento de cabeza, anteroversión de la pelvis. Estos aspectos se relacionan con una mayor seguridad en el contacto y en los apoyos, al realizar un equilibrio dinámico reducido (Nakamura, 1997; Gazzellini et al., 2016).

Uysal et al. (2010) mencionan que se deben de mejorar las capacidades físicas y motrices como la flexibilidad, la fuerza, y el equilibrio que intervienen en el control motriz. Muchas de estas deficiencias se podrían deber a la ausencia de estimulación en edades tempranas, a la falta de libertad en los movimientos y del conocimiento de la orientación espacial. Además, Ray et al. (2007), afirman que un impedimento, más allá de la ausencia de visión, es la falta de actividad física en las PDV.

En el estudio de da Silva et al. (2018), se evidencia que las PDV que son físicamente activas no muestran diferencias en los aspectos medidos de la caminata (velocidad, zancada, ritmo), al compararlos con personas de visión normal.

Por otro lado, se recomienda el análisis integral de aspectos relacionados a factores sociales, culturales, económicos y emocionales, porque estas variables podrían también afectar la independencia y la autonomía de las PDV.

\section{CONCLUSIÓN}

Se concluye que existe poca evidencia de estudios experimentales que incluyan caminar como variable; por otro lado, se muestra que las PDV, presentan diferencias significativas al compararlos con personas con visión normal. En donde se detectaron marchas atípicas en PDV, en algunos casos mostraron grandes rangos de 
movimientos articular, como el tobillo, o por el contrario, movimientos pequeños cómo en la cabeza y la pelvis, también, mayor flexión de tronco y cabeza, indicando poca economía y técnica en los movimientos, por lo que se realizan de forma forzada, ejerciendo mayor estrés muscular y óseo (Gazzellini et al., 2016; Patla, 1997; Timmis et al., 2014). Otros aspectos como el frenado, la propulsión, la fuerza de reacción en el suelo de las PDV no son congruentes en los estudios (Mason et al., 2005.; Ray et al., 2007; Timmis et al., 2014), por lo que se sugiere como variables en estudios futuros.

Una limitación de la revisión fue que casi todos los estudios fueron descriptivos.

\section{Recomendaciones}

Es importante realizar investigaciones que:

- Comparen parámetros funcionales con el control motor, y como se puede mejorar con intervenciones.

- Valoren padecimientos asociados a las adaptaciones hechas por las PDV, como por ejemplo, si padecen de dolores de espalda, rodillas, tobillos, entre otros.

- Relacionen los patrones de marcha con los acortamientos musculares.

- Muestren el efecto del ejercicio sobre los patrones de la marcha en PDV.

- Involucren un grupo multidisciplinario de profesiones.

- Tomen en cuenta diferentes grupos etarios.

- Tomen en cuenta aspectos socioculturales, para tener mayor efecto en las intervenciones.

- Tengan diferencias o semejanzas entre sexo para tener recomendaciones más específicas.

Es crucial la valoración en situaciones reales de las PDV, para poder brindar herramientas con las que se defiendan en su roll de independiente, y de accesibilidad.

\section{REFERENCIAS}

Agostini, V., Sbrollini, A., Cavallini, C., Busso, A., Pignata, G., \& Knaflitz, M. (2016). The role of central vision in posture: Postural sway adaptations in Stargardt patients. Gait \& Posture, 43, 233-238. https://doi.org/10.101 6/j.gaitpost.2015.10.003

Agudelo, A. I., Briñez, T.J., Guarrín, V., Ruiz, J. P.y Zapata, M. C. (2013). Marcha: descripción, métodos, herramientas de evaluación y parámetros de normalidad reportados en la literatura. CES Movimiento y Salud 1(1) 29-43. Recuperado de http://revistas.ces.edu.co/index.php/movimientoysalud/article/view/2481

Alan, W. D. (1991). Psychological correlates of walking speed in the visually impaired. Ergonomics, 34(1), 91-102. h ttps://doi.org/10.1080/00140139108967291

Aydoğ, E., Aydoğ, S., Çakci, A., \& Doral, M. (2006). Dynamic Postural Stability in Blind Athletes Using The Biodex Stability System. International Journal of Sports Medicine, 27(5), 415-418. https://doi.org/10.1055/s-2005-86 5777

Chen, C.C., \& Lin, S.Y. (2011). The impact of rope jumping exercise on physical fitness of visually impaired students. Research in Developmental Disabilities, 32(1), 25-29. https://doi.org/10.1016/j.ridd.2010.08.010

Chen, E. W., Fu, A. S. N., Chan, K. M., \& Tsang, W. W. N. (2012). The effects of Tai Chi on the balance control of elderly persons with visual impairment: a randomised clinical trial. Age and Ageing, 41(2), 254-259. https:/ /doi.org/10.1093/ageing/afr 146

Clark-Carter, D. D., Heyes, A. D., \& Howarth, C. I. (1986). The efficiency and walking speed of visually impaired people. Ergonomics, 29(6), 779-789. https://doi.org/10.1080/00140138608968314 
MHSALUd, ISSN: 1659-097X, 17(1), ENERo-Junio, 2020, pP 1-10

ALemán-RAMíreZ

da Silva, E. S., Fischer, G., da Rosa, R. G., Schons, P., Teixeira, L. B. T., Hoogkamer, W., \& Peyré-Tartaruga, L. A. (2018). Gait and functionality of individuals with visual impairment who participate in sports. Gait \& Posture, 62, 355-358. https://doi.org/10.1016/j.gaitpost.2018.03.049

Gazzellini, S., Lispi, M. L., Castelli, E., Trombetti, A., Carniel, S., Vasco, G., ... Petrarca, M. (2016). The impact of vision on the dynamic characteristics of the gait: strategies in children with blindness. Experimental Brain Research, 234(9), 2619-2627. https://doi.org/10.1007/s00221-016-4666-9

Hallemans, A., Ortibus, E., Meire, F., \& Aerts, P. (2010). Low vision affects dynamic stability of gait. Gait \& Posture, 32(4), 547-551. https://doi.org/10.1016/j.gaitpost.2010.07.018

Knutzen, K., Hamill, J. \& Bates, B. (1985). Ambulatory characteristics of the visually disabled. Human Movement Science 4, 55-66. https://doi.org/10.1016/0167-9457(85)90023-5

Kobberling, G., Jankowski, L. W., \& Leger, L. (1989). Energy Cost of Locomotion in Blind Adolescents. Adapted Physical Activity Quarterly, 6(1), 58-67. https://doi.org/10.1123/apaq.6.1.58

Larsson, L., \& Frändin, K. (2006). Body Awareness and Dance-Based Training for Persons with Acquired Blindness -Effects on Balance and Gait Speed. Visual Impairment Research, 8(1-2), 25-40. https://doi.org/10.1080/13 882350600964667

Mason, S. J., Legge, G. E., \& Kallie, C. S. (2005). Variability in the Length and Frequency of Steps of Sighted and Visually Impaired Walkers. Journal of visual impairment and blindness. 99 (15) 741-754. PMID: 20198108

Moher, D., Liberti, A., Telzlaff, J. y Altman, D. (2009). Preferred Reporting Items for Systematic Reviews and MetaAnalyses: the PRISMA Statement, Annals of Internal Medicine, 151(4). 264-270. https://doi.org/10.1371/jou rnal.pmed.1000097

Nakamura, T. (1997). Quantitative analysis of gait in the visually impaired. Disability and Rehabilitation, 19(5), 194-197. https://doi.org/10.3109/09638289709166526

Parreira, R. B., Grecco, L. A. C., \& Oliveira, C. S. (2017). Postural control in blind individuals: A systematic review. Gait \& Posture, 57, 161-167. https://doi.org/10.1016/j.gaitpost.2017.06.008

Patla, A. E. (1997). Understanding the roles of vision in the control of human locomotion. Gait \& Posture, 5(1), 54-69. https://doi.org/10.1016/S0966-6362(96)01109-5

Ramsey, V., Blasch, B., Akio, K. y Jonhson, B. (1999). A Biomechanical Evaluation of Visually Impaired Persons Gait and Long-Cane Machanics. Journal of Rehabilitation Research and Development, 36 (4), 323-332. PMID: 10678455

Ray, C., Horvat, M., Williams, M., \& Blasch, B. (2007). Kinetic Movement Analysis in Adults with Vision Loss. Adapted Physical Activity Quarterly, 24(3), 209-217. https://doi.org/10.1123/apaq.24.3.209

Reynard, F., \& Terrier, P. (2015). Role of visual input in the control of dynamic balance: variability and instability of gait in treadmill walking while blindfolded. Experimental Brain Research, 233(4), 1031-1040. https://doi.org/ $10.1007 / \mathrm{s} 00221-014-4177-5$

Saibene, F., \& Minetti, A. E. (2003). Biomechanical and physiological aspects of legged locomotion in humans. European Journal of Applied Physiology, 88(4), 297-316. https://doi.org/10.1007/s00421-002-0654-9

Theodorou, A., \& Skordilis, E. (2012). Evaluating the Approach Run of Class F1 1 Visually Impaired Athletes in Triple and Long Jumps. Perceptual and Motor Skills, 114(2), 595-609. https://doi.org/10.2466/05.15.27.PMS.114.2 $.595-609$

Timmis, M. A., Scarfe, A. C., Tabrett, D. R., \& Pardhan, S. (2014). Kinematic analysis of step ascent among patients with central visual field loss. Gait \& Posture, 39(1), 252-257. https://doi.org/10.1016/j.gaitpost.2013.07.115

Uysal, S. A., Erden, Z., Akbayrak, T., \& Demirtürk, F. (2010). Comparison of Balance and Gait in Visually or Hearing Impaired Children. Perceptual and Motor Skills, 111(1), 71-80. https://doi.org/10.2466/10.11.15.25.PMS.11 1.4.71-80

White, U. E., Black, A. A., Wood, J. M., \& Delbaere, K. (2015). Fear of Falling in Vision Impairment. Optometry and Vision Science, 92(6), 730-735. Retrieved from https://www.ncbi.nlm.nih.gov/pubmed/25930978 
Żyłka, J., Lach, U., \& Rutkowska, I. (2013). Functional Balance Assessment With Pediatric Balance Scale in Girls With Visual Impairment: Pediatric Physical Therapy, 25(4), 460-466. https://doi.org/10.1097/PEP.0b013e31 $829 \mathrm{ddbc} 8$

CC BY-NC-ND 